CASE REPORT

\title{
Eosinophilic ascites: a diagnostic challenge
}

\author{
Hesham Khalil, Moby Joseph
}

Department of

Gastroenterology, Great

Western Hospitals NHS

Foundation Trust, Swindon, UK

\section{Correspondence to}

Dr Hesham Khalil,

hesham.khalil@gwh.nhs.uk

Accepted 17 August 2016

\section{SUMMARY}

Eosinophilic ascites is a rare feature of eosinophilic gastroenteritis. We would like to highlight this increasingly recognised diagnosis in a case of unexplained ascites. We present a challenging case of a woman aged 25 years who presented with nausea, vomiting, diarrhoea, generalised abdominal pain and swelling 8-week following delivery of her first baby. Her symptoms were primarily aggravated by eating, and she had also noticed postprandial itching and self-limiting generalised rash. She had a strong history of atopy. Physical examination revealed abdominal tenderness and distension with shifting dullness. Urticarial skin rash was noted on the face, neck, chest and abdomen. Routine biochemistry was normal apart from peripheral eosinophilia. Imaging confirmed moderate ascites. Diagnostic paracentesis showed exudative ascites with numerous eosinophils. Histology of the upper and lower gastrointestinal tract showed infiltration of the oesophageogastroduodenal and rectosigmoid mucosa with eosinophils. The patient significantly improved following a course of steroids and six-food elimination diet.

\section{BACKGROUND}

Eosinophilic ascites (EA) is a rare feature of eosinophilic gastroenteritis (EGE) characterised by eosinophilic infiltration of the gastrointestinal (GI) tract with serosal involvement. The aetiology of EGE/EA is not well recognised. It often presents as a case of unexplained ascites on top of a group of nonspecific symptoms, such as nausea, vomiting, diarrhoea, abdominal pain, itching and rash. It has a chronic relapsing course. Peripheral eosinophilia is a commonly associated feature and a marker for the disease activity. Its presentation can mimic many GI and systemic disorders and can be easily misdiagnosed.

\section{CASE PRESENTATION}

A Caucasian woman aged 25 years presented 8 -week postpartum following an uneventful pregnancy of her first baby, with abdominal pain, nausea, vomiting, diarrhoea and progressive generalised abdominal swelling. She opened her bowels 4-5 times per day passing type 7 stool with no blood or mucous. Her symptoms were primarily aggravated by eating, and she had also noticed postprandial itching and self-limiting generalised rash.

The patient had a history of asthma, Hay fever and allergic rhinitis. She previously developed anaphylactic shock in response to intravenous iodinebased radio-contrast agent. During her hospital stay, she developed contact dermatitis in response to plasters. There was no history of liver or heart disease. Her regular medications included only inhalers for asthma. The patient was a non-smoker and denied alcohol ingestion. She had family history of food allergies where two of her brothers are allergic to blackcurrant and barley.

Physical examination, including cardiovascular and rheumatological assessments, was unremarkable. Focused abdominal examination revealed distended, lax and generally tender abdomen with rebound tenderness. The patient had positive shifting dullness. No palpable organs or masses were detected. No jaundice or any stigmata of chronic liver disease were found. Urticarial skin rash was noted on the face, neck, chest and abdomen (figure 1).

\section{INVESTIGATIONS}

Blood investigations showed high eosinophilic count at $2.67 \times 10 / \mathrm{mL}(30 \%)$ in an otherwise normal full blood count. Blood film confirmed marked eosinophilia with no immature cells or

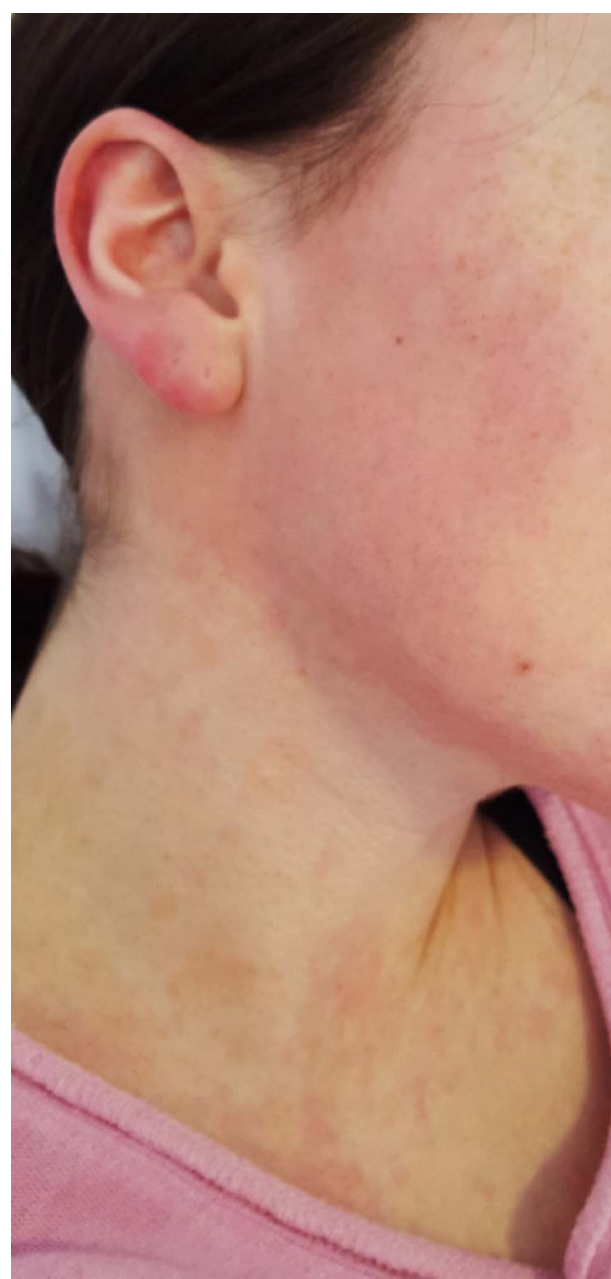

Figure 1 Skin rash on the chest, neck and face. 
blasts. Serum electrolytes, liver function tests, coagulation screen and inflammatory markers were within normal ranges. Full immunological, vasculitic and coeliac screen were all negative. Immunoglobulin electrophoresis, including $\operatorname{IgE}$ level, was normal. Viral screen, including hepatitis B virus, hepatitis C virus, HIV, Epstein-Barr virus and cytomegalovirus, was negative. Thyroid function, serum amylase and serum ACE levels were normal. Urinary metanephrines and hydroxyindoleacetic acid were also normal. Parasitic infestation was ruled out by the absence of parasitic cysts or ova in stool in addition to negative ELISA test for Toxocara and Strongyloides. Furthermore, urine and stool cultures were negative.

Ultrasonography showed normal echotexture of the liver with ascites around the upper border of the liver. The portal and hepatic venous flow was normal. Non-contrast CT abdomen and pelvis revealed free intraperitoneal fluid with normal liver, kidney, spleen and pancreas. No peritoneal nodularity or bowel abnormality had been identified (figure 2A, B).

Diagnostic paracentesis revealed cloudy straw-coloured fluid. Chemical analysis showed exudative ascites with serum-acites albumin gradient (SAAG) ratio of 8. Amylase, triglycerides, glucose and lactate dehydrogenase in the ascetic fluid were normal. Ascetic fluid bacterial and tuberculous enrichment cultures did not grow any organisms. The white cell count in the ascetic fluid was $2558 \times 10^{6} / \mathrm{L}$. Cytological examination revealed numerous eosinophils, scattered histiocytes, neutrophils, lymphocytes and occasional normal mesothelial cells. No evidence of malignant cells was noted (figure $3 \mathrm{~A}, \mathrm{~B}$ ).

Oesophageogastroduodenoscopy demonstrated erythema and micro-abscesses in the antrum and body of the stomach (figure 4). Mucosal biopsies were obtained, which were consistent with EGE (figure 5A-C). Histopathological examination revealed infiltration of the upper GI tract mucosa with eosinophils predominantly in the midoesophagus, gastric antrum and duodenum; reaching up to 30 per high power field in the second part of the duodenum. Flexible sigmoidoscopy showed a normal looking mucosa up to the distal transverse colon. Mucosal biopsies were consistent with eosinophilic colitis. High power field microscopy showed predominant stromal eosinophils (reaching up to 25 per HPF) with quite prominent degranulation of eosinophilic cytoplasm.

\section{DIFFERENTIAL DIAGNOSIS}

- Parasitic infestations (eg, Strongyloides stercoralis, Toxocara canis)

- Spontaneous bacterial peritonitis

- Abdominal tuberculosis

- Peritoneal sarcoidosis

- Carcinoid syndrome

- Pheochromocytoma

- Hypothyroidism

- Chronic pancreatitis

- Crohn's disease

- Vasculitis (eg, Churgstrauss syndrome)

- Systemic autoimmune diseases

- HIV

- Systemic hypersensitivity reactions

- Hypereosinophilic syndrome

- Malignancy (eg, ovarian cancer, peritoneal cancer)

- Peritoneal dialysis

\section{TREATMENT}

After confirming the diagnosis, a combination therapy of oral steroids and six-food elimination diet (SFED) was started. Reducing regime of oral prednisolone $30 \mathrm{mg} /$ day was followed
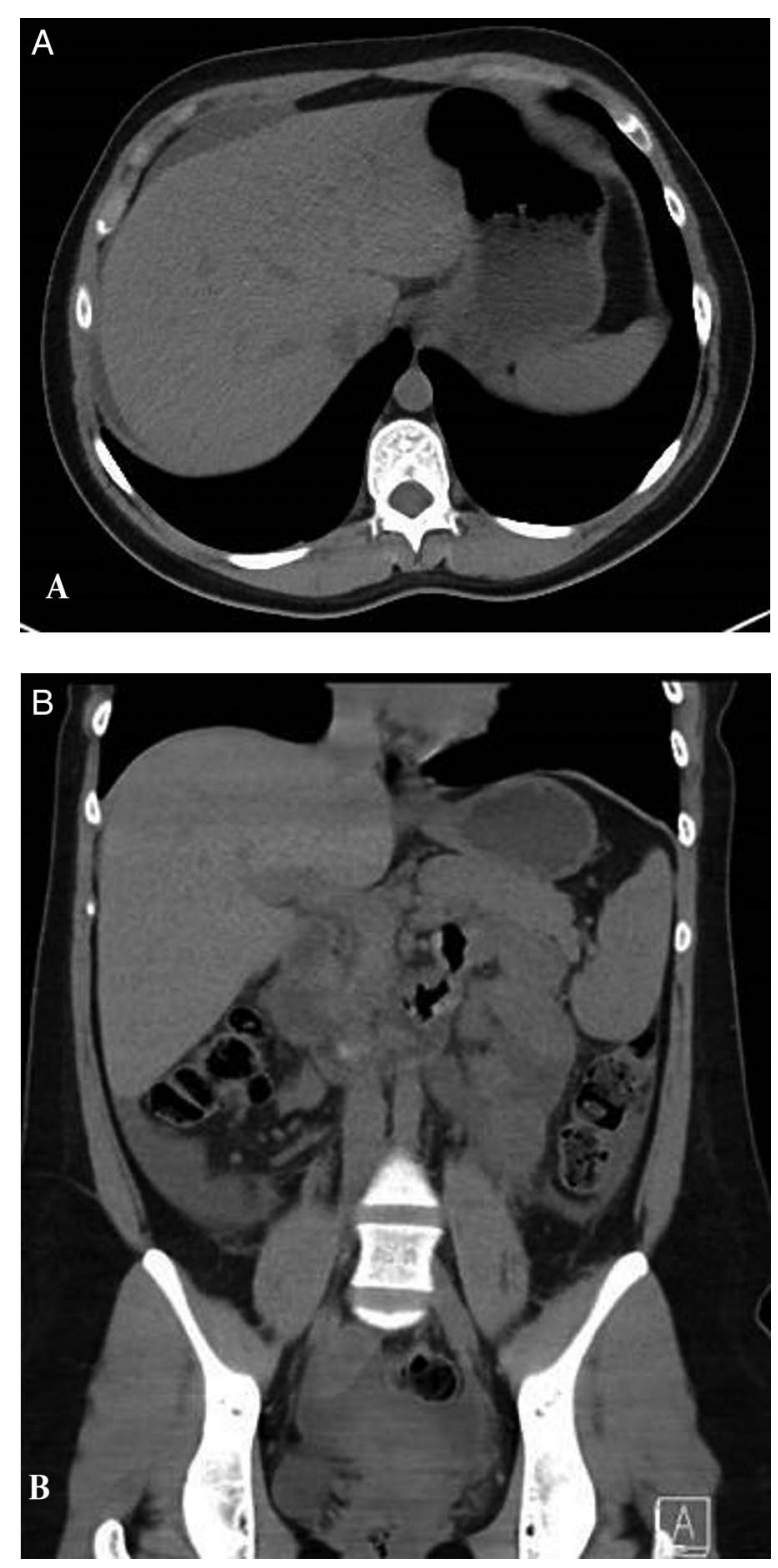

Figure 2 (A, B) Non-contrast $\mathrm{CT}$ abdomen and pelvis demonstrating the degree of ascites.

by a low maintenance dose. The SFED eliminates the most likely food allergens, including milk, egg, soy, wheat, peanuts/ tree nuts and fish/shellfish.

\section{OUTCOME AND FOLLOW-UP}

The patient showed dramatic improvement on oral steroids and SFED. On the clinical grounds, her symptoms had resolved within 3 days from the initiation of steroids. Biochemically, the peripheral eosinophilic count had fallen down to normal level on day 2 steroids (figure 6). Radiologically, ascites had markedly improved with only a trace amount of $6 \mathrm{~mm}$ in the pelvis on day 7 steroids. Eight weeks later, the patient was found to be clinically, biochemically and radiologically stable.

\section{DISCUSSION}

EGE is an uncommon condition characterised by sporadic or diffuse eosinophilic infiltration of the GI tract with subsequent degranulation and destruction of the GI wall. It was first described by Kaijser in $1937 .{ }^{1}$ EGE seems to be a restrictive 

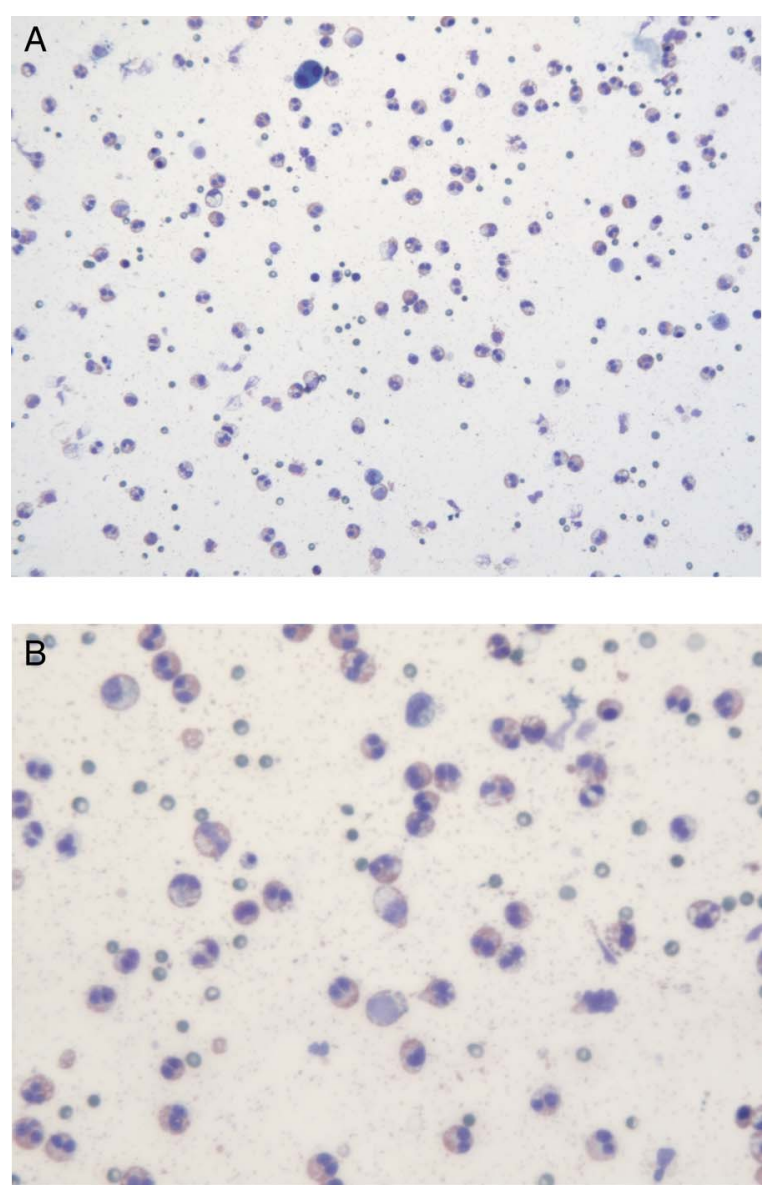

Figure 3 (A, B) Ascetic fluid examination showing numerous eosinophils, scattered histiocytes, neutrophils, lymphocytes and occasional normal mesothelial cells. A, B are magnified 100x and $200 x$, respectively.

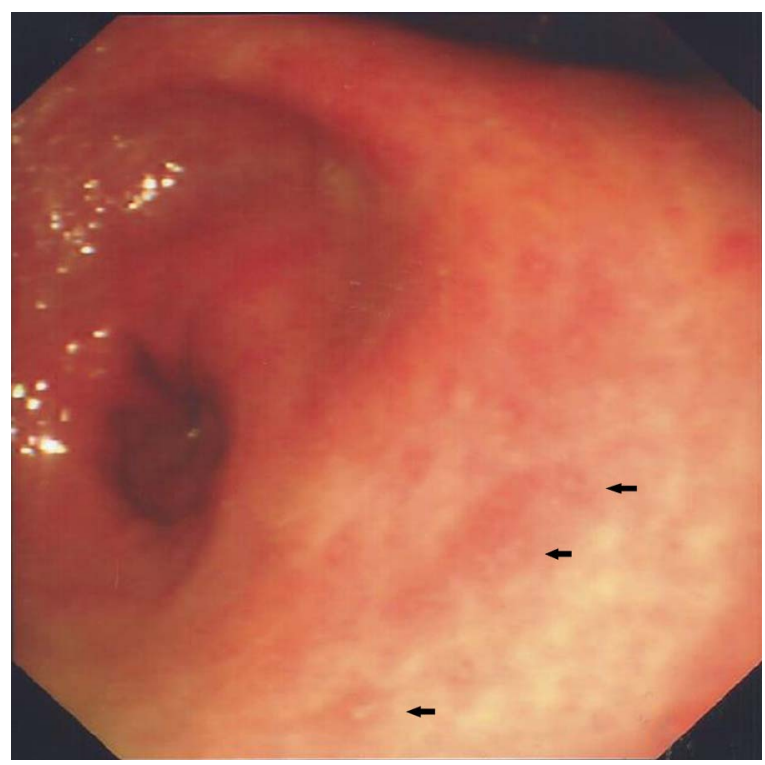

Figure 4 Gastroscopic appearance demonstrating erythema and micro-abscesses (arrows) in the body and antrum.

misnomer as the entire GI system can be infiltrated; from the oesophagus to the colon and even the biliary tract. ${ }^{2}$ Eosinophilic infiltration has specific predilection to the stomach and the small bowel. ${ }^{3}$ Concurrent involvement of the oesophagus and/or colon may exist; however, the latter is considered
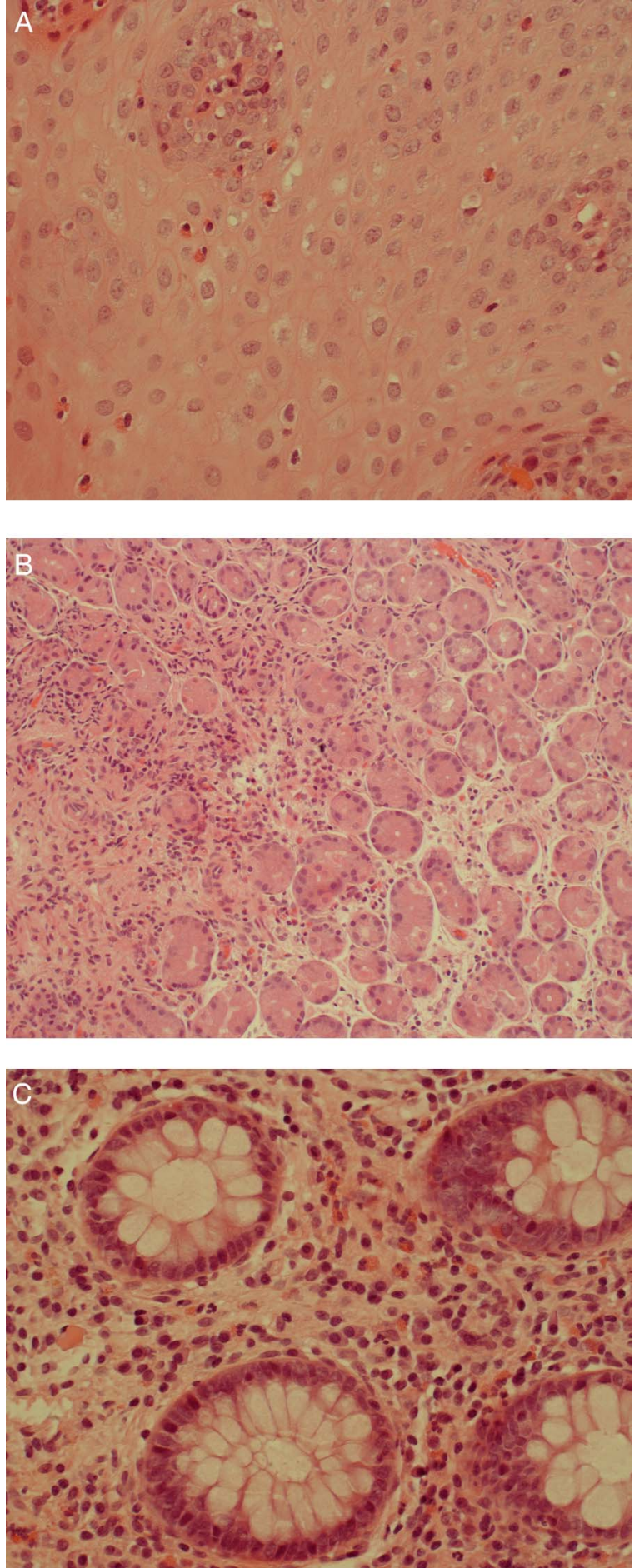

Figure 5 A, B and C demonstrate eosinophilic mucosal infiltration of the oesophagus, stomach and colon, respectively.

uncommon. The fact that eosinophilic infiltration can predominate in different layers of the gut wall was first introduced by Klein et al in 1970. Accordingly, patients were classified into three groups: predominant mucosal, muscular or subserosal disease. Mucosal-predominant disease, the most common form of presentation, is defined as mucosal infiltration by eosinophils, in the absence of muscle layer infiltration, signs of GI obstruction or importantly EA. It may present with nausea, vomiting, diarrhoea, abdominal pain, anaemia or protein-loosing enteropathy. Muscular-predominant disease, defined as eosinophilic 


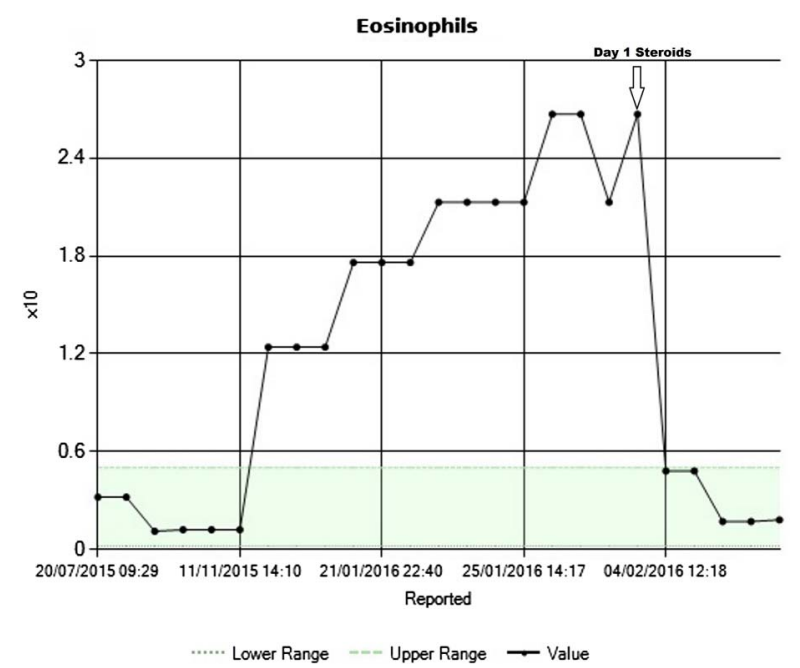

Figure 6 A graph illustrating the effect of steroid therapy on the peripheral eosinophilic count. The number of eosinophils has significantly dropped following the first day of steroid therapy (arrow) reaching a normal range after 2 days.

infiltration of the tunica muscularis with no evidence of EA, frequently causes stricture formation and bowel obstruction. Subserosal-predominant disease, the rarest form of presentation, is characterised by the presence of eosinophilic infiltration of the GI system and EA. ${ }^{4}$ Patients with transmucosal disease and ascites were included in the subserosal group. ${ }^{5}$ Despite decades of ongoing research, the aetiology and pathogenesis of EGE are still uncertain. However, it is often associated with atopic conditions such as asthma, Hay fever, or drug or food allergy. ${ }^{6}$ Pregnancy and childbirth can sometimes trigger the flare of EGE. ${ }^{7}$ We here report a case of subserosal EGE involving the entire GI tract that developed 8-week postpartum. It is very difficult to ascertain involvement of the muscular layer in the absence of radiological evidence of GI wall thickening and lack of full thickness biopsy. However, as the mucosal and serosal layers were affected, it is also possible that the muscular layer was involved with lack of predominance.

Unlike the majority of other GI conditions, EGE does not have a clear confirmatory test and lacks unified criteria for diagnosis. As yet, the diagnosis of EGE is based on high level of clinical suspicion supported by consistent histopathological findings. It is paramount to highlight that when the diagnosis is suspected, other causes of unexplained ascites associated with GI symptoms should be excluded. EA has a wide range of differential diagnoses. Strongyloides and Toxocara infestation may mimic the presentation of EGE/EA, and must be ruled out, ${ }^{8}$ since initiating steroid therapy in those patients carries high risk of disseminated infection and potentially death. The parasitic infestation and EGE may increase the serum Ig-E level; ${ }^{9}$ however, this was normal in our case. Cytological evaluation is a cornerstone in the diagnosis of EA. Endoscopic evaluation may show a wide range of non-specific changes depending on the distribution and depth of tissue involvement. It can vary from macroscopically normal mucosa to marked erythematous changes, with erosions, microabcesses, inflammatory polyps, mucosal ulceration and stricture formation. ${ }^{10}$ Histology often demonstrates heavy eosinophilic infiltration of the GI mucosa with degranulation; however, biopsies may lack the predominance of eosinophils in patients with patchy disease. ${ }^{5}$ In some cases of EA, the diagnosis might be challenging, due to the absence of essential diagnostic characteristics. It is more commonly occurring in the subserosal type of EGE, in which the clinical picture might be lacking systemic eosinophilia or even eosinophilic infiltration of the GI mucosa. In those cases, laparoscopic serosal biopsies are mandatory to confirm the diagnosis. However, predominant eosinophils in the ascetic fluid and fast response to steroids should guide towards the diagnosis. ${ }^{11}$ Alternatively, ultrasound-guided percutaneous gastric and small intestinal biopsies can provide diagnostic evidence for subserosal and muscular types of EGE. ${ }^{12}$

Corticosteroids and elimination diets are the mainstay of therapy. Steroids play an important role in the induction of remission guided by clinical response and peripheral eosinophilic count. Of note, the vast majority of patients with subserosal type of EGE are fast steroid responders. Nevertheless, a low maintenance dose of oral steroids is usually required to maintain remission. Alternatively, anti-allergic agents, such as mast cell stabilisers, antihistamines, leukotriene receptor antagonists or immunosuppressant may act as steroid-sparing drugs. ${ }^{13}$ Elimination diets aim to identify and eliminate the offending food allergen from diet.

In conclusion, EGE/EA is an easily missed diagnosis which should be kept in mind, especially in young atopic individuals presenting in the postpartum period with ascites of undetermined aetiology and peripheral eosinophilia. After excluding malignancy, the predominance of eosinophils in the ascetic fluid and significant response to steroids should provide enough evidence to confirm the diagnosis in most cases.

\section{Learning points}

- Eosinophilic ascites (EA) is a rare condition, which should be considered in all cases of unexplained ascites.

- EA is not uncommon in atopic individuals in the postpartum period.

- Peripheral eosinophilia is a good marker for the disease activity in EA.

Acknowledgements Dr HK would like to express his deepest gratitude and appreciation to Dr. Nahed El Hefnawy for her outstanding care and endless support. He would also like to deeply thank Dr Mohamed Farag for his massive efforts and supreme support.

Contributors HK and MJ were involved in the patients care. HK carried out the literature search and wrote the first manuscript. Editing was performed by MJ. All the authors approved the final manuscript. I certify that neither this manuscript nor one with substantially similar content under my authorship has been published or is being considered for publication elsewhere. I have access to any data on which the manuscript is based and will provide such data on request to the editors or their assignees. I have given final approval of the submitted manuscript for which I take public responsibility for whole content.

Competing interests None declared.

Patient consent Obtained.

Provenance and peer review Not commissioned; externally peer reviewed.

\section{REFERENCES}

1 Whitaker IS, Gulati A, McDaid JO, et al. Eosinophilic gastroenteritis presenting as obstructive jaundice. Eur J Gastroenterol Hepatol 2004;16:407-9.

2 Schoonbroodt D, Horsmans Y, Laka A, et al. Eosinophilic gastroenteritis presenting with colitis and cholangitis. Dig Dis Sci 1995;40:308-14.

3 Khan S, Orenstein SR. Eosinophilic gastroenteritis: epidemiology, diagnosis and management. Paediatr Drugs 2002;4:563-70.

4 Klein NC, Hargrove RL, Sleisenger MH, et al. Eosinophilic gastroenteritis. Medicine (Baltimore) 1970;49:299-319.

5 Talley NJ, Shorter RG, Phillips SF, et al. Eosinophilic gastroenteritis: a clinicopathological study of patients with disease of the mucosa, muscle layer, and subserosal tissues. Gut 1990;31:54-8. 
6 Lee $\mathrm{CM}$, Changchien $\mathrm{CS}$, Chen PC, et al. Eosinophilic gastroenteritis: 10 years experience. Am J Gastroenterol 1993;88:70-4.

7 Ogasa M, Nakamura $Y$, Sanai $H$, et al. A case of pregnancy associated hypereosinophilia with hyperpermeability symptoms. GynecolObstet Invest 2006:62:14-16.

8 Lawate P, Singh SP. Eosinophilic ascites due to Strongyloides stercoralis. Trop Gastroenterol 2005;26:91-2.

9 Rothenberg ME. Eosinophilic gastrointestinal disorders (EGID). J Allergy Clin/mmunol 2004; 113:11-28.
10 Fox VL. Eosinophilic esophagitis: endoscopic findings. Gastrointest Endosc Clin N Am 2008;18:45-57, viii.

11 To Y, Ogawa C, Otomo M, et al. A case of eosinophilic gastroenteritis complicated with ileus and ascites collection. Arerugi 1999:48:50-5.

12 Sleiman I, Villanacci V, Pelizzari G, et al. Ultrasound guided percutaneous fineneedle biopsy in a case of eosinophilic gastroenteritis. J Med Liban 1998;46:100-2.

13 Urek MC, Kujundzić M, Banić $M$, et al. Leukotriene receptor antagonists as potential steroid sparing agents in a patient with serosal eosinophilic gastroenteritis. Gut 2006;55:1363-4.

Copyright 2016 BMJ Publishing Group. All rights reserved. For permission to reuse any of this content visit http://group.bmj.com/group/rights-licensing/permissions.

BMJ Case Report Fellows may re-use this article for personal use and teaching without any further permission.

Become a Fellow of BMJ Case Reports today and you can:

- Submit as many cases as you like

- Enjoy fast sympathetic peer review and rapid publication of accepted articles

- Access all the published articles

- Re-use any of the published material for personal use and teaching without further permission

For information on Institutional Fellowships contact consortiasales@bmjgroup.com

Visit casereports.bmj.com for more articles like this and to become a Fellow 